\title{
A signature-based approach to quantify soil moisture dynamics under contrasting land-uses
}

\author{
Ryoko Araki ${ }^{1}$, Flora Branger ${ }^{2}$, Inge Wiekenkamp ${ }^{3}$, and Hilary McMillan ${ }^{1}$ \\ ${ }^{1}$ San Diego State University \\ ${ }^{2}$ Irstea Centre de Lyon-Villeurbanne \\ ${ }^{3}$ Helmholtz Centre Potsdam German Research Centre for Geosciences
}

December 21, 2021

\begin{abstract}
Soil moisture signatures provide a promising solution to overcome the difficulty of evaluating soil moisture dynamics in hydrologic models. Soil moisture signatures are metrics that quantify the dynamic aspects of soil moisture timeseries and enable processbased model evaluations. To date, soil moisture signatures have been tested only under limited land-use types. In this study, we explore soil moisture signatures' ability to discriminate different dynamics among contrasting land-uses. We applied a set of nine soil moisture signatures to datasets from six in-situ soil moisture networks worldwide. The dataset covered a range of land-use types, including forested and deforested areas, shallow groundwater areas, wetlands, urban areas, grazed areas, and cropland areas. Our set of signatures characterized soil moisture dynamics at three temporal scales: event, season, and a complete timeseries. Statistical assessment of extracted signatures showed that (1) event-based signatures can distinguish different dynamics for all the land-uses, (2) season-based signatures can distinguish different dynamics for some types of land-uses (deforested vs. forested, urban vs. greenspace, and cropped vs. grazed vs. grassland contrasts), (3) timeseries-based signatures can distinguish different dynamics for some types of land-uses (deforested vs. forested, urban vs. greenspace, shallow vs. deep groundwater, wetland vs. non-wetland, and cropped vs. grazed vs. grassland contrasts). Further, we compared signature-based process interpretations against literature knowledge; event-based and timeseries-based signatures generally matched well with previous process understandings from literature, but season-based signatures did not. This study will be a useful guideline for understanding how catchment-scale soil moisture dynamics in various land-uses can be described using a standardized set of hydrologically relevant metrics.
\end{abstract}

\section{Hosted file}

HYP-21-0457-R1_Proof_hi.pdf available at https://authorea.com/users/421061/articles/527226-asignature-based-approach-to-quantify-soil-moisture-dynamics-under-contrasting-land-uses 


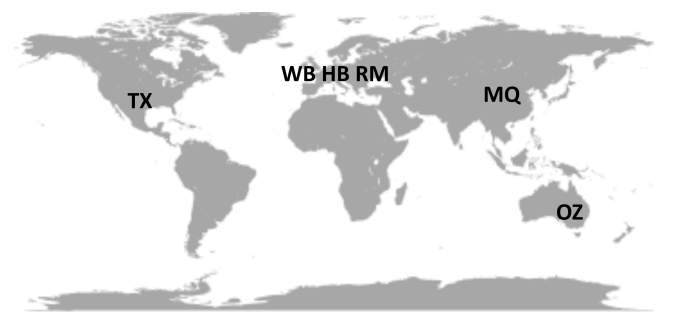

\section{Legend \\ Study site (abbreviation)}

Land-use

Number of sensors and installation depth Observation period

- Watershed / network extent

- Elevation contour (Interval varies. $2 \mathrm{~m}$ for WB, $200 \mathrm{~m}$ for HB, RM, TX, $900 \mathrm{~m}$ for MQ, $2000 \mathrm{~m}$ for OZ)

O Sensors (Undisturbed land-use)

- Sensors (Disturbed land-use)

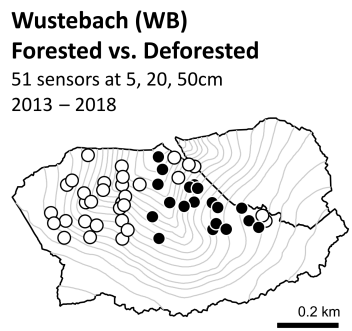

Hamburg (HB)

Greenspace vs. Housing

Raam (RM)

15 sensors at $5,10,40,80,160 \mathrm{~cm}$

Raam (RM)

water

$2010-2016$

2016- 2017
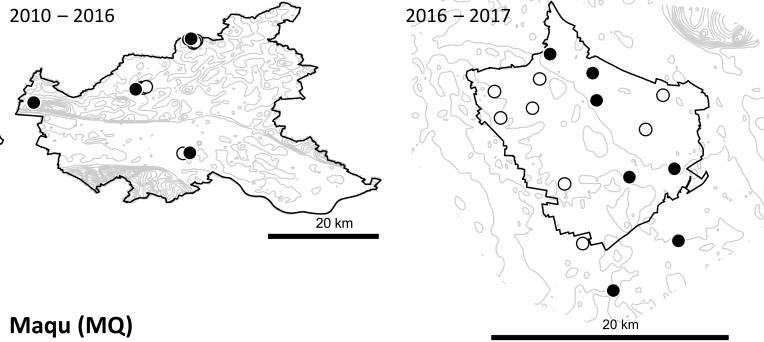

Ungrazed vs. Grazed

38 sensors at $5,10,20,50 \mathrm{~cm}$ $2014-2019$

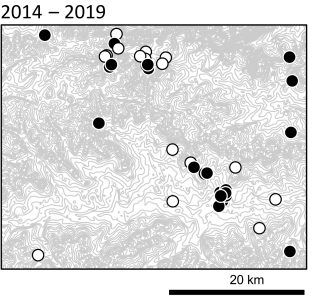

Maqu (MQ)

Non-wetland vs. Wetland

20 sensors at $5,10,20,40,80 \mathrm{~cm}$ $2008-2010$

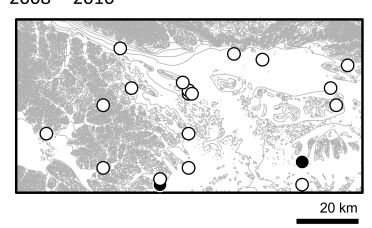

Oznet (OZ)

Grass vs. Grazed vs. Crop

38 sensors at $3,4,15,45,75 \mathrm{~cm}$

$2001-2019$

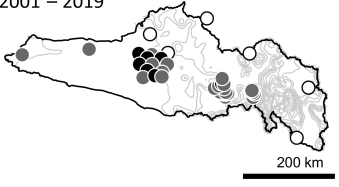

(a)

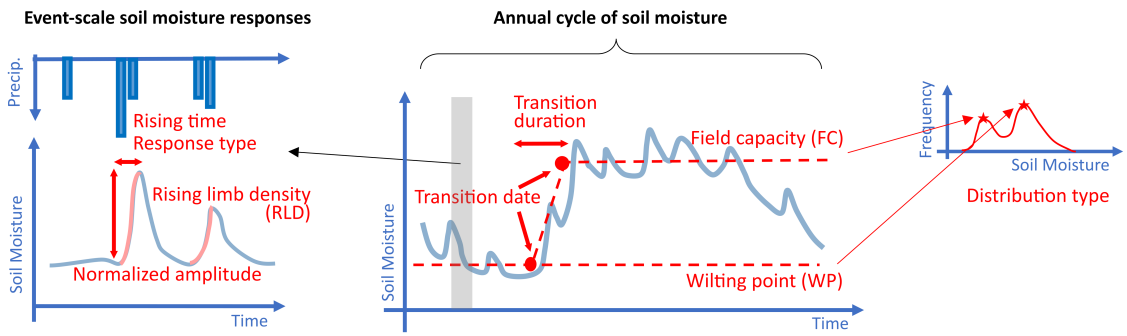

(b)

\begin{tabular}{|c|c|c|c|c|}
\hline & & \multicolumn{3}{|l|}{ Timescale } \\
\hline & & Event & Season & Complete timeseries \\
\hline \multirow[t]{4}{*}{ Dynamics } & Shape & $\begin{array}{l}\text { Rising limb density } \\
\text { (Sawicz et al., 2011) }\end{array}$ & - & $\begin{array}{l}\text { Distribution type } \\
\text { (Branger \& McMillan, 2019; } \\
\text { Graham and Lin, 2012) }\end{array}$ \\
\hline & Timing & $\begin{array}{l}\text { Response type } \\
\text { (Graham and Lin, 2012; } \\
\text { Wiekenkamp et al., 2016) }\end{array}$ & $\begin{array}{l}\text { Transition date } \\
\text { (Branger \& McMillan, 2019) }\end{array}$ & - \\
\hline & Speed & $\begin{array}{l}\text { Rising time } \\
\text { (Branger \& McMillan 2019) }\end{array}$ & $\begin{array}{l}\text { Transition duration } \\
\text { (Branger \& McMillan, 2019) }\end{array}$ & - \\
\hline & Magnitude & $\begin{array}{l}\text { Normalized amplitude } \\
\text { (Branger \& McMillan, 2019) }\end{array}$ & - & $\begin{array}{l}\text { Field capacity } \\
\text { Wilting point } \\
\text { (Branger \& McMillan, 2019; } \\
\text { Chandler et al., 2017) }\end{array}$ \\
\hline
\end{tabular}




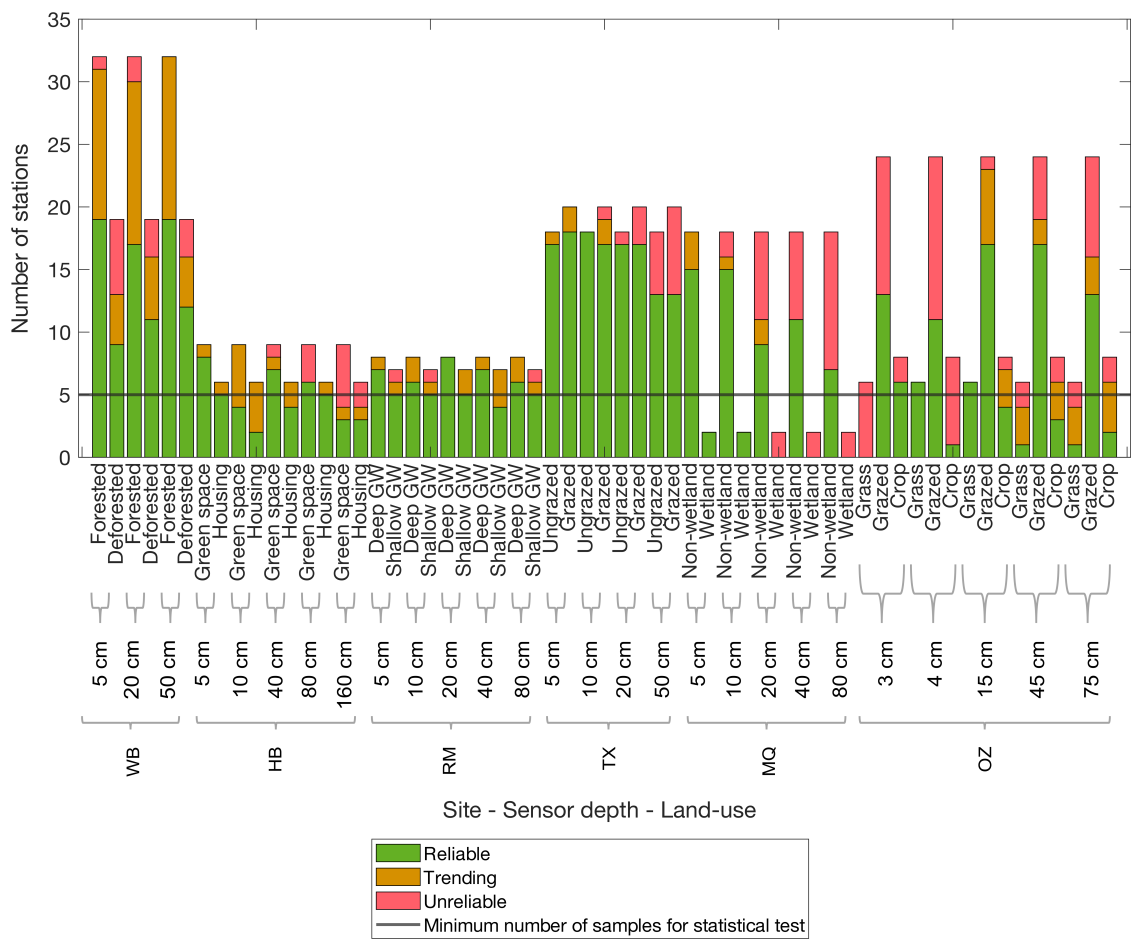


Event-based signatures
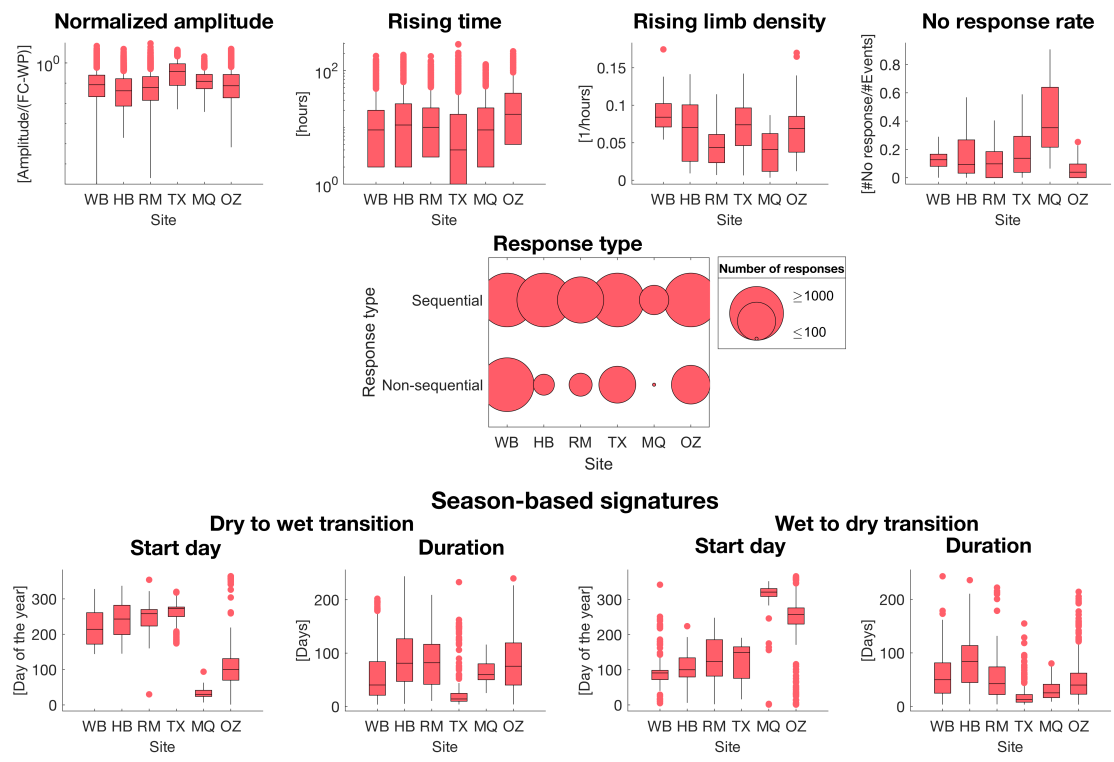

Timeseries-based signatures
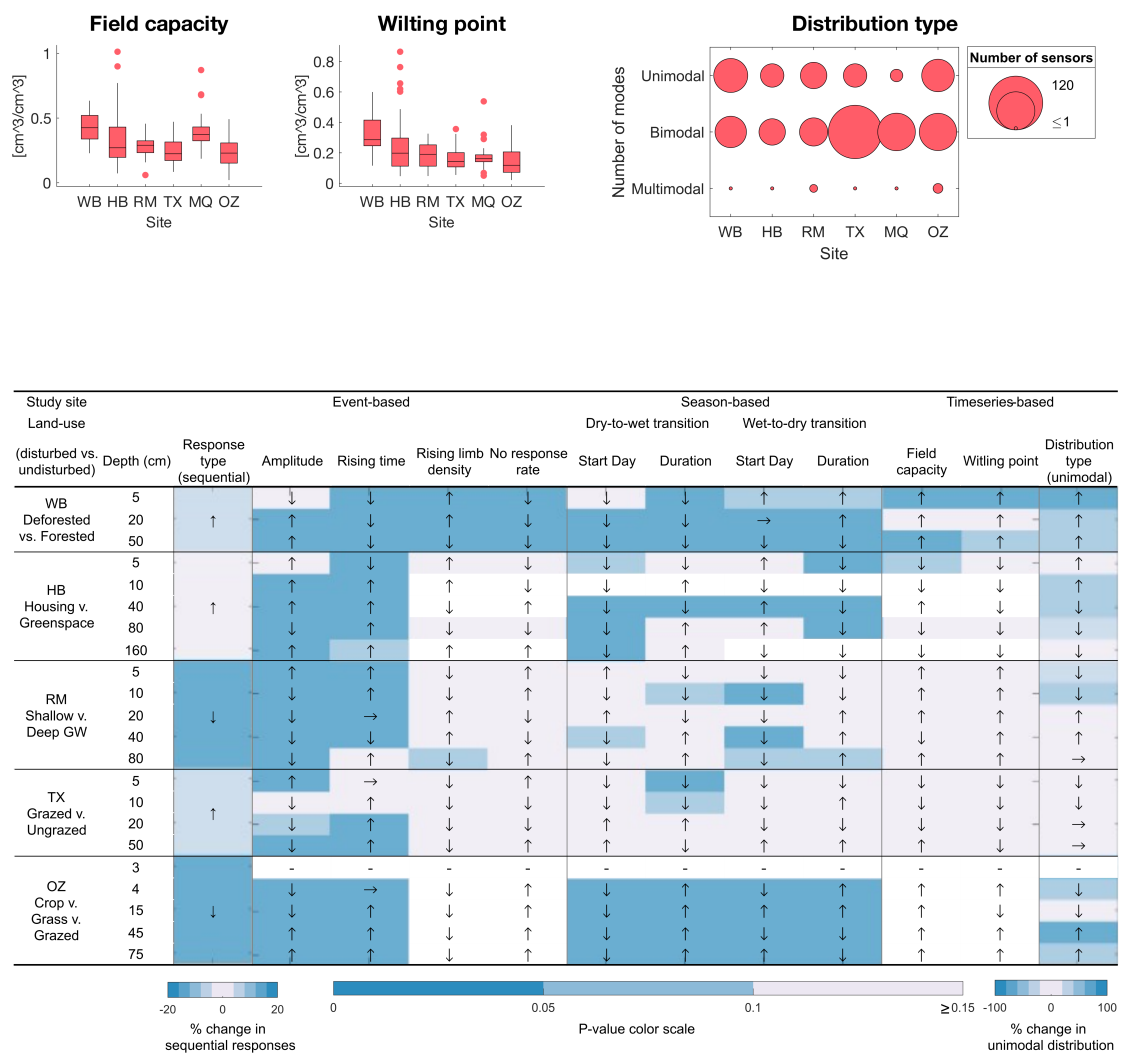
Event-based signatures
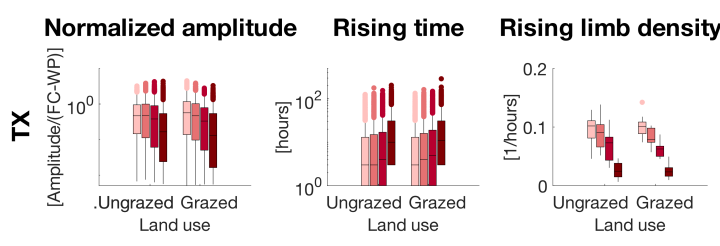

No response rate

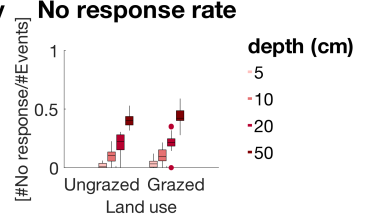

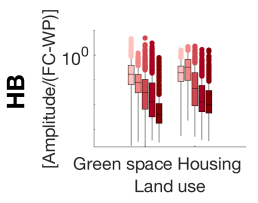
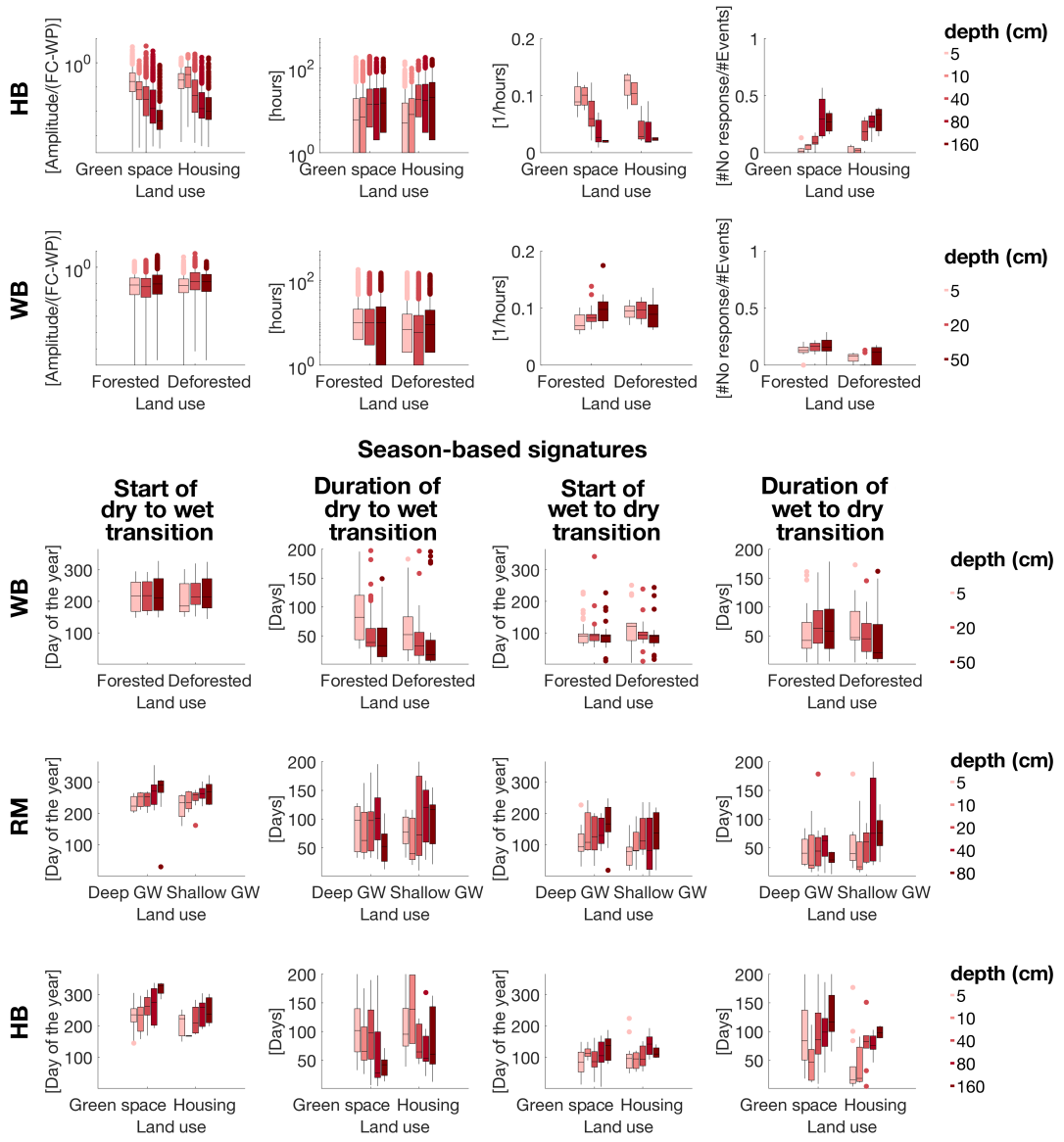
Timeseries-based signatures
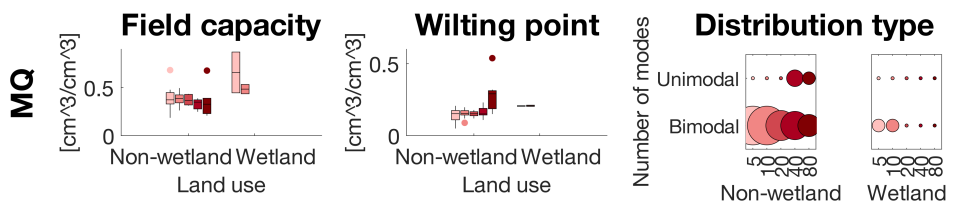

depth (cm)
-5
-10
-20
-40
-80
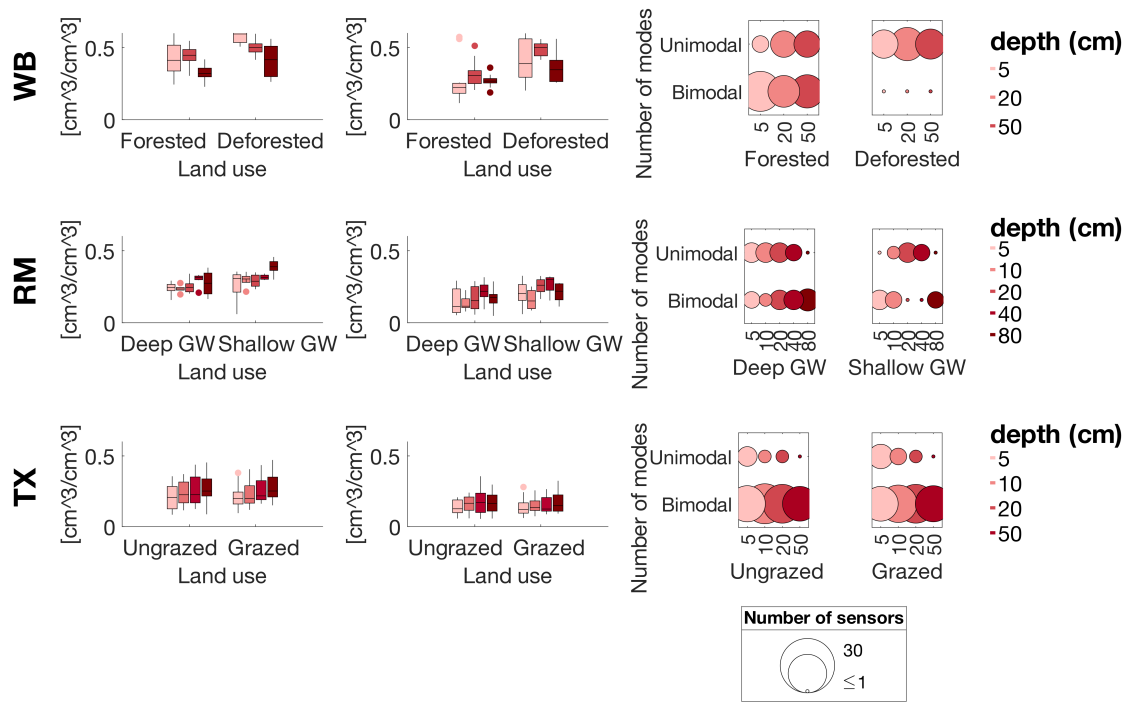


\begin{tabular}{|c|c|c|c|c|c|c|c|c|c|c|c|c|c|}
\hline & \multicolumn{12}{|c|}{ Signatures } \\
\hline & & \multicolumn{5}{|c|}{ Event-based } & \multicolumn{4}{|c|}{ Season-based } & \multicolumn{3}{|c|}{ Timeseries-based } \\
\hline & & \multirow{2}{*}{\begin{tabular}{|c|} 
Response \\
type (\% of \\
sequential) \\
\end{tabular}} & \multirow[b]{2}{*}{ Amplitude } & \multirow{2}{*}{\begin{tabular}{|c|} 
Rising \\
time \\
\end{tabular}} & \multirow{2}{*}{$\begin{array}{c}\text { Rising limb } \\
\text { density }\end{array}$} & \multirow{2}{*}{\begin{tabular}{|c|}
$\begin{array}{c}\text { No- } \\
\text { response } \\
\text { rate }\end{array}$ \\
\end{tabular}} & \multicolumn{2}{|c|}{ Dry-to-wet transition } & \multicolumn{2}{|c|}{ Wet-to-dry transition } & \multirow{2}{*}{$\begin{array}{c}\text { Field } \\
\text { capacity }\end{array}$} & \multirow{2}{*}{$\begin{array}{c}\text { Wilting } \\
\text { point }\end{array}$} & \multirow{2}{*}{$\begin{array}{l}\text { Distribution } \\
\text { type }(\% \text { of } \\
\text { unimodal) }\end{array}$} \\
\hline & & & & & & & Start Day & Duration & Start Day & Duration & & & \\
\hline \multirow{3}{*}{$\begin{array}{c}\text { Wüstebach } \\
\text { Deforested } \\
\text { vs. } \\
\text { Forested }\end{array}$} & $\begin{array}{c}\text { Expected } \\
\text { process }\end{array}$ & \multicolumn{5}{|c|}{ 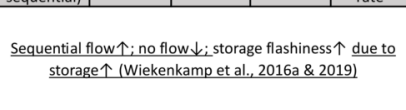 } & \multicolumn{4}{|c|}{$\begin{array}{c}\text { Earlier transition due to interception } \downarrow \& \text { rain } \\
\text { rate } \uparrow \& \text { root depth } \downarrow \text { (Wiekenkamp et al.. } \\
\underline{2016 \mathrm{~b} ; \text { Laio, 2002); closer to transition }} \\
\text { threshold due to storage } \uparrow \text { (Detty \& McGuire, } \\
\text { 2010) }\end{array}$} & \multicolumn{3}{|c|}{$\begin{array}{l}\frac{\text { Storage } \uparrow \text { due to transpiration } \downarrow}{\text { \& interception } \downarrow \text { (Wiekenkamp }} \\
\text { et al., 2016a) }\end{array}$} \\
\hline & $\begin{array}{l}\text { Expected } \\
\text { signature }\end{array}$ & 1 & $\uparrow$ & $\downarrow$ & $\uparrow$ & $\underline{\perp}$ & $\downarrow$ & $\downarrow$ & $\downarrow$ & $\downarrow$ & 1 & 1 & 1 \\
\hline & $\begin{array}{l}\text { Observed } \\
\text { signature }\end{array}$ & $\uparrow$ & $\uparrow$ & $\downarrow$ & $\begin{array}{c}\text { shallow } \uparrow \\
\text { deep } \downarrow\end{array}$ & $\downarrow$ & $\downarrow$ & $\downarrow$ & $\rightarrow$ & $\begin{array}{c}\text { shallow } \uparrow \\
\text { deep } \downarrow\end{array}$ & $\uparrow$ & $\uparrow$ & $\uparrow$ \\
\hline \multirow{3}{*}{$\begin{array}{l}\text { Hamburg } \\
\text { Housing } \\
\text { vs. } \\
\text { Urban }\end{array}$} & $\begin{array}{c}\text { Expected } \\
\text { process }\end{array}$ & \multicolumn{5}{|c|}{$\begin{array}{l}\text { Vertical infiltration }>\text { overland flow due to surface sealing } \\
\text { (Scalenghe \& Ajmone-Marsan, 2009; Ziegler et al., 2001); } \\
\text { storage flashiness } \downarrow \text { due to storage } \downarrow\end{array}$} & \multicolumn{4}{|c|}{\begin{tabular}{|c|} 
Delayed transition due to surface sealing \& \\
rain rate $\downarrow$ (Laio, 2002); stagnant water \& \\
rapid drainage due to construction waste \\
(Wiesner et al., 2016); less close to transition \\
threshold due to storage $\downarrow$ (Detty \& McGuire, \\
2010)
\end{tabular}} & \multicolumn{3}{|c|}{$\begin{array}{c}\text { Storage } \downarrow \& \text { GW table } \downarrow \text { due to } \\
\text { infiltration } \downarrow \text { (Scalenghe \& } \\
\text { Ajmone-Marsan, 2009; Wiesner } \\
\text { et al., 2016) }\end{array}$} \\
\hline & $\begin{array}{l}\text { Expected } \\
\text { signature }\end{array}$ & $\uparrow$ & $\begin{array}{c}\text { shallow } \uparrow \\
\text { deep } \downarrow\end{array}$ & $\begin{array}{c}\text { shallow } \downarrow \\
\text { deep } \uparrow\end{array}$ & $\begin{array}{l}\text { shallow } \uparrow \\
\text { deep } \downarrow\end{array}$ & $\begin{array}{c}\text { shallow } \downarrow \\
\text { deep } \uparrow\end{array}$ & $\uparrow$ & $\uparrow$ & 工 & $\downarrow$ or $\uparrow$ & 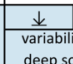 & 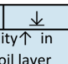 & deep $\downarrow$ \\
\hline & $\begin{array}{l}\text { Observed } \\
\text { signature }\end{array}$ & $\uparrow$ & $\begin{array}{c}\text { shallow } \uparrow \\
\text { deep } \uparrow\end{array}$ & $\begin{array}{c}\text { shallow } \downarrow \\
\text { deep } \uparrow\end{array}$ & $\begin{array}{l}\text { shallow } \uparrow \\
\text { deep } \downarrow\end{array}$ & $\begin{array}{c}\text { shallow } \downarrow \\
\text { deep } \uparrow\end{array}$ & $\downarrow$ & $\rightarrow$ & $\rightarrow$ & $\downarrow$ & 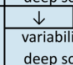 & 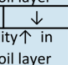 & $\begin{array}{c}\text { shallow } \uparrow \\
\text { deep } \downarrow\end{array}$ \\
\hline $\begin{array}{l}\text { Raam } \\
\text { Shallow } \\
\text { vs. }\end{array}$ & $\begin{array}{c}\text { Expected } \\
\text { process }\end{array}$ & less variable s & $\begin{array}{l}\text { Vertical infil } \\
\text { soil moistur } \\
\&\end{array}$ & $\begin{array}{l}\text { Itration } \rightarrow \text { la } \\
\text { re due to nea } \\
\text { Bras, 2021) }\end{array}$ & $\begin{array}{l}\text { teral flow; } \\
\text { ar-saturated }\end{array}$ & soil (Soylu & $\begin{array}{l}\text { Earlier trar } \\
\text { Macho \& } \\
\text { threshold } \\
\end{array}$ & $\begin{array}{l}\text { nsition due } \\
\text { Fan, 2012); } \\
\text { due to stora } \\
20\end{array}$ & $\begin{array}{l}\text { to shallow } G \\
\text { more close to } \\
\text { ge } \uparrow \text { (Detty } \\
\text { b10) }\end{array}$ & $\begin{array}{l}\text { GW (Miguez- } \\
\text { to transition } \\
\text { \& McGuire, }\end{array}$ & $\underline{\text { Storage }}$ & $\begin{array}{l}\text { 1 due to } \\
\text { sa et al., } 2 \text { C } \\
\text { Bras, } 20\end{array}$ & $\begin{array}{l}\text { capillary rise } \\
\frac{118 \mathrm{~b}}{20)} \text {; Soylu \& }\end{array}$ \\
\hline $\begin{array}{c}\text { Deep } \\
\text { groundwater }\end{array}$ & \begin{tabular}{|l} 
Expected \\
signature
\end{tabular} & $\downarrow$ & $\downarrow$ & $\downarrow$ & $\rightarrow$ & $\rightarrow$ & $\downarrow$ & $\downarrow$ & $\downarrow$ & $\downarrow$ & 1 & 1 & $\uparrow$ \\
\hline (GW) & $\begin{array}{l}\text { Observed } \\
\text { signature }\end{array}$ & $\downarrow$ & $\downarrow$ & $\uparrow$ & $\rightarrow$ & $\rightarrow$ & $\rightarrow$ & $\rightarrow$ & $\downarrow$ & $\rightarrow$ & $\rightarrow$ & $\rightarrow$ & $\begin{array}{l}\text { shallow } \downarrow \\
\text { deep } \uparrow\end{array}$ \\
\hline Texas & $\begin{array}{l}\text { Expected } \\
\text { process }\end{array}$ & $\begin{array}{l}\text { Vertical infi } \\
\text { (Woodruff \& }\end{array}$ & $\begin{array}{l}\text { filtration }>0 \\
\& \text { Wilding, } 2 \\
\end{array}$ & $\begin{array}{l}\text { overland flor } \\
\text { :008; Alaoui } \\
\text { al., 2001) }\end{array}$ & $\begin{array}{l}\text { w due to cor } \\
\text { et al., 2018; }\end{array}$ & mpaction & $\begin{array}{r}\text { Less clo } \\
\text { stora }\end{array}$ & $\begin{array}{l}\text { se to transit } \\
\text { ge } \downarrow \text { (Detty }\end{array}$ & $\begin{array}{l}\text { tion threshol } \\
\text { \& McGuire, }\end{array}$ & $\begin{array}{l}\text { old due to } \\
\text {,2010) }\end{array}$ & $\begin{aligned} \text { Storage } \\
\text { (Borma } \\
\text { Selass }\end{aligned}$ & $\begin{array}{l}\text { e due to } \\
\text { ann \& Klaa } \\
\text { sie \& Ayan }\end{array}$ & $\begin{array}{l}\text { compaction } \\
\text { ssen, 2008; } \\
\text { na, 2013) }\end{array}$ \\
\hline $\begin{array}{l}\text { Grazed } \\
\text { vs. }\end{array}$ & $\begin{array}{l}\text { Expected } \\
\text { signature }\end{array}$ & $\uparrow$ & \begin{tabular}{|c|} 
shallow $\uparrow$ \\
deep $\downarrow$ \\
\end{tabular} & $\begin{array}{c}\text { shallow } \downarrow \\
\text { deep } \uparrow\end{array}$ & $\begin{array}{c}\text { shallow } \uparrow \\
\text { deep } \downarrow\end{array}$ & \begin{tabular}{|c|} 
shallow $\downarrow$ \\
deep $\uparrow$
\end{tabular} & $\rightarrow$ & $\uparrow$ & $\rightarrow$ & $\uparrow$ & $\downarrow$ & $\downarrow$ & $\downarrow$ \\
\hline & $\begin{array}{l}\text { Observed } \\
\text { signature }\end{array}$ & $\uparrow$ & $\begin{array}{c}\text { shallow } \uparrow \\
\text { deep } \downarrow\end{array}$ & $\uparrow$ & $\rightarrow$ & $\rightarrow$ & $\rightarrow$ & $\downarrow$ & $\rightarrow$ & $\rightarrow$ & $\rightarrow$ & $\rightarrow$ & $\rightarrow$ \\
\hline $\begin{array}{c}\text { Maqu } \\
\text { Wetland }\end{array}$ & $\begin{array}{l}\text { Expected } \\
\text { processes }\end{array}$ & $\begin{array}{r}\text { Less variable } \\
\& B r\end{array}$ & $\begin{array}{l}\text { soil moistur } \\
\text { ras, 2021); le }\end{array}$ & $\begin{array}{l}\text { re due to ne } \\
\text { ess response }\end{array}$ & $\begin{array}{l}\text { ar-saturated } \\
\text { es while froz }\end{array}$ & $\begin{array}{l}\text { soil (Soylu } \\
\text { en }\end{array}$ & $\begin{array}{r}\text { Seasonz } \\
\text { growth d } \\
\text { Freeze } \\
\text { del }\end{array}$ & $\begin{array}{l}\text { al transition } \\
\text { lo not chang } \\
\text { /thaw proc } \\
\text { ayed due to }\end{array}$ & $\begin{array}{l}\text { timing of ve } \\
\text { ze (Dente et } \\
\text { ess takes lon } \\
\text { heat capaci }\end{array}$ & $\begin{array}{l}\text { egetation } \\
\text { tal., 2012)i } \\
\text { nger and } \\
\text { city } \uparrow\end{array}$ & $\frac{\underline{\text { Storage }}}{\frac{\mathrm{matte}}{\mathrm{Hu}}}$ & $\begin{array}{l}\text { T due to } \\
\text { r (Dente e } \\
\text { idson et al }\end{array}$ & $\begin{array}{l}\frac{\text { soil organic }}{\text { tal., 2012; }} \\
\text {, 2014) }\end{array}$ \\
\hline $\begin{array}{c}\text { vs. } \\
\text { Non-wetland }\end{array}$ & $\begin{array}{l}\text { Expected } \\
\text { signature }\end{array}$ & $\downarrow$ & $\downarrow$ & $\downarrow$ & $\downarrow$ & $\uparrow$ & $\exists$ or $\uparrow$ & $\exists$ or $\uparrow$ & $\exists$ or $\uparrow$ & $\exists$ or $\uparrow$ & 1 & 1 & $\uparrow$ \\
\hline & \begin{tabular}{|l} 
Observed \\
signature
\end{tabular} & $\begin{array}{c}\text { Not enough } \\
\text { data }\end{array}$ & $\rightarrow$ & $\rightarrow$ & $\downarrow$ & $\uparrow$ & $\rightarrow$ & $\rightarrow$ & $\rightarrow$ & $\rightarrow$ & $\uparrow$ & $\uparrow$ & $\uparrow$ \\
\hline $\begin{array}{c}\text { Oznet } \\
\text { Crop vs. } \\
\text { Grazed vs. }\end{array}$ & $\begin{array}{c}\text { Expected } \\
\text { process }\end{array}$ & $\begin{array}{l}\text { Vertical infi } \\
\text { (Alaoui et al. }\end{array}$ & $\begin{array}{r}\text { filtration }>0 \\
\text { I., 2018; Zieg } \\
\uparrow d u\end{array}$ & $\begin{array}{l}\text { overland-flo } \\
\text { ler et al., } 20 \\
\text { ue to storage }\end{array}$ & $\begin{array}{l}\text { w due to cor } \\
\text { o1); storage } \\
\mathrm{e} \uparrow\end{array}$ & $\begin{array}{l}\text { mpaction } \\
\text { flashiness }\end{array}$ & $\begin{array}{r}\text { More clo } \\
\text { storage } \uparrow \\
\text { wet peri }\end{array}$ & $\begin{array}{l}\text { ose to trans } \\
\text { (Detty \& Mc } \\
\text { iod due to it } \\
\underline{2 C}\end{array}$ & $\begin{array}{l}\text { ition thresho } \\
\text { Guire, 2010) } \\
\text { rigation (Sm } \\
\text { D12) }\end{array}$ & $\begin{array}{l}\text { old due to } \\
\text { p); extended } \\
\text { mith et al.., }\end{array}$ & $\begin{array}{r}\text { Storage } \\
\text { (Borma } \\
\text { Selass } \\
\text { Storag } \\
\text { Smith e } \\
\end{array}$ & $\begin{array}{l}\downarrow \text { due to } \\
\text { ann \& Klaa } \\
\text { sie \& Ayan } \\
\text { se } \uparrow \text { due to } \\
\text { tal., } 2012 \\
\text { al., } 201\end{array}$ & $\begin{array}{l}\text { compaction } \\
\text { ssen, 2008; } \\
\text { na, 2013); } \\
\text { i irrigation } \\
\text {; Lawston et } \\
\text { 7) }\end{array}$ \\
\hline Grass & $\begin{array}{l}\text { Expected } \\
\text { signature }\end{array}$ & $\uparrow$ & $\begin{array}{l}\text { shallow } \uparrow \\
\text { deep } \downarrow\end{array}$ & \begin{tabular}{|c|} 
shallow $\downarrow$ \\
deep $\uparrow$
\end{tabular} & $\begin{array}{c}\text { shallow } \uparrow \\
\text { deep } \downarrow\end{array}$ & \begin{tabular}{|c|} 
shallow $\downarrow$ \\
deep $\uparrow$
\end{tabular} & $\downarrow$ & $\downarrow$ & $\uparrow$ & $\downarrow$ & $\downarrow$ or $\uparrow$ & $\downarrow$ or $\uparrow$ & $\downarrow$ or $\uparrow$ \\
\hline & $\begin{array}{l}\begin{array}{l}\text { Observed } \\
\text { signature }\end{array} \\
\end{array}$ & $\downarrow$ & $\begin{array}{c}\text { shallow } \downarrow \\
\text { deep } \uparrow\end{array}$ & $\uparrow$ & $\downarrow$ & $\uparrow$ & $\downarrow$ & $\uparrow$ & $\begin{array}{l}\text { mixed } \\
\uparrow \& \downarrow\end{array}$ & \begin{tabular}{|c|} 
shallow $\uparrow$ \\
deep $\downarrow$
\end{tabular} & $\uparrow$ & $\uparrow$ & $\begin{array}{c}\text { shallow } \downarrow \\
\text { deep } \uparrow\end{array}$ \\
\hline
\end{tabular}

Observed signature matches with literature interpretation

Observed signature does not match with literature interpretation 


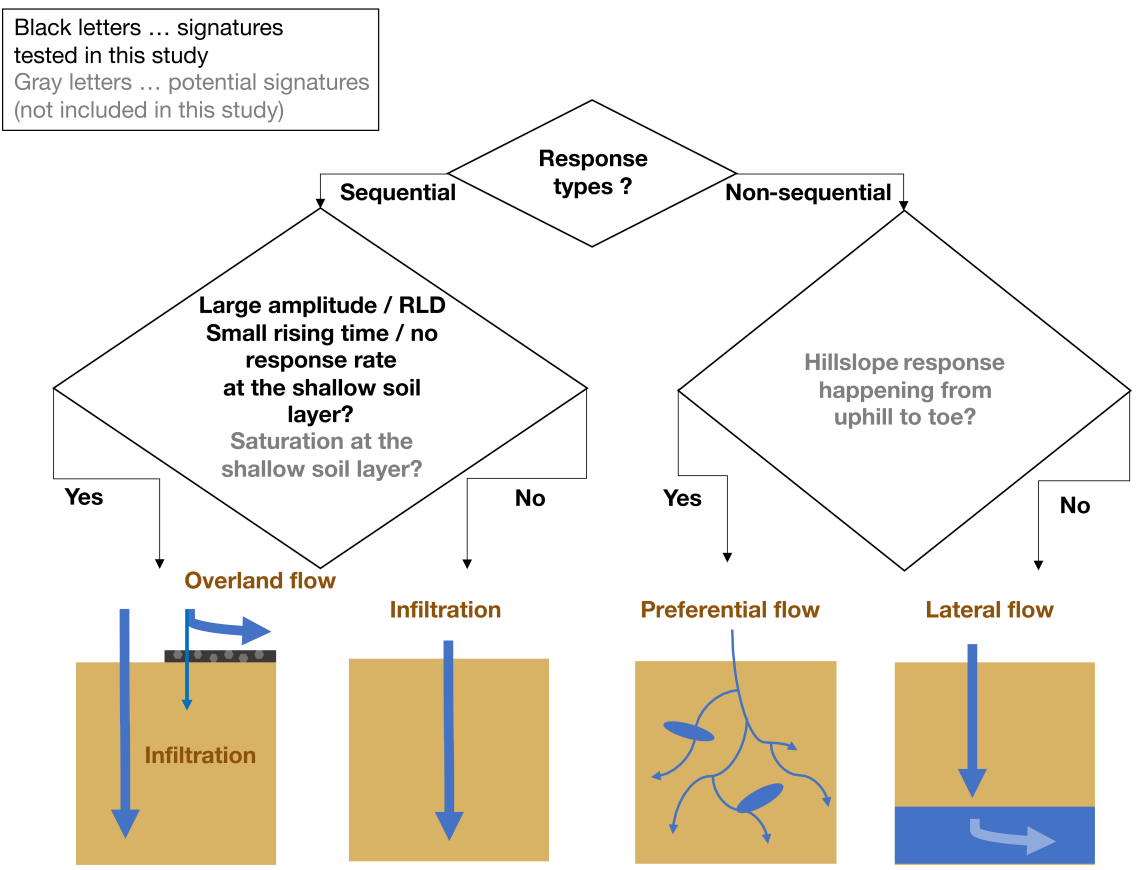

\section{Hosted file}

tab1_studysites.docx available at https://authorea.com/users/421061/articles/527226-asignature-based-approach-to-quantify-soil-moisture-dynamics-under-contrasting-land-uses 